\title{
Prevalence and associated factors of pistol grip deformity in Japanese local residents
}

\author{
Takaya Taniguchi ${ }^{1 凶}$, Teiji Harada ${ }^{1,8}$, Toshiko lidaka ${ }^{2,8}{ }^{2}$ Hiroshi Hashizume ${ }^{1}$, \\ Wataru Taniguchi ${ }^{1}$, Hiroyuki Oka ${ }^{3}$, Yoshiki Asai $^{1}$, Shigeyuki Muraki ${ }^{2}$, Toru Akune ${ }^{4}$, \\ Kozo Nakamura ${ }^{5}$, Hiroshi Kawaguchi ${ }^{6}$, Munehito Yoshida ${ }^{1}$, Sakae Tanaka ${ }^{7}$, \\ Hiroshi Yamada $^{1,8}$ \& Noriko Yoshimura ${ }^{2,8}$
}

Pistol grip deformity (PGD) may be the main factor in femoroacetabular impingement development. This study aimed to clarify the epidemiological indices and factors related to PGD in Japanese people. This population-based cohort study included 1575 local Japanese residents. PGD, center edge angle, and joint space width were measured radiographically. We investigated the relationship between PGD and spino-pelvic parameters. Factors associated with PGD were examined using multiple logistic regression analysis, with the presence/absence of $P G D$ as an objective variable, and sex, age, body mass index (BMI), and the presence/absence of hip pain or spino-pelvic parameters as explanatory variables. In the entire cohort, 4.9\% (10.6\% men, 2.1\% women) had PGD on at least one side. A trend was observed between PGD and increasing age in both men and women (men: $p<0.0001$, women: $\mathrm{p}=0.0004$ ). No relationship was observed between $\mathrm{PGD}$ and hip pain (risk ratio 1.0 [95\% confidence interval 0.97-1.03]). Factors significantly associated with PGD were age, sex, and BMI in the multivariate model. Acquired factors may be related to PGD in Japanese people as the PGD prevalence increased with age and PGD was not significantly associated with hip pain. This study provides new insights into the etiology and clinical significance of PGD.

Patients with primary osteoarthritis of the hip often experience swelling in the lateral part of the femoral headneck junction. This occurrence is termed pistol grip deformity (PGD) ${ }^{1,2}$. PGD has attracted significant attention since several authors have reported the disease concept of femoroacetabular impingement (FAI $)^{3-6}$. There are three types of hip impingements: cam, pincer, and combined. The cam-type is due to an abnormal morphology of the junction between the femoral head and neck, while the pincer type is due to an excessively deep acetabular floor. PGD is presumably the main factor of cam-type FAI in European countries and the United States ${ }^{7}$. In recent years, many reports have suggested FAI as an etiology of hip pain and primary/idiopathic osteoarthritis ${ }^{8,9}$. PGD is considered a congenital deformity ${ }^{10}$. In addition, several studies have suggested an association between PGD and spino-pelvic parameters, including pelvic incidence ${ }^{11,12}$.

Owing to technical advances in magnetic resonance imaging (MRI) diagnostics and hip arthroscopy, the presence and pathology of FAI have begun to attract attention in Japan, where secondary osteoarthritis of the hip due to acetabular dysplasia is common ${ }^{13}$. However, the epidemiology of PGD remains unclear, and the association between PGD and hip pain or primary osteoarthritis has not been elucidated among the Japanese population. Further, the relationship between PGD and spino-pelvic parameters is unknown in this population.

This study aimed to clarify the prevalence of PGD in different age groups and sexes and elucidate related factors, including hip pain, osteoarthritis, and spino-pelvic parameters, in a Japanese population.

\footnotetext{
${ }^{1}$ Department of Orthopaedic Surgery, Wakayama Medical University, 811-1 Kimiidera, Wakayama, Wakayama, Japan. ${ }^{2}$ Department of Preventive Medicine for Locomotive Organ Disorders, 22nd Century Medical and Research Center, The University of Tokyo, Hongo, Bunkyo-ku, Tokyo, Japan. ${ }^{3}$ Department of Medical Research and Management for Musculoskeletal Pain, 22nd Century Medical and Research Center, Faculty of Medicine, The University of Tokyo, Hongo, Bunkyo-ku, Tokyo, Japan. ${ }^{4}$ Rehabilitation Services Bureau, National Rehabilitation Center for Persons with Disabilities, 4-1 Namiki, Tokorozawa, Saitama, Japan. ${ }^{5}$ Department of Orthopaedic Surgery, Towa Hospital, Towa, Adachi-ku, Tokyo, Japan. ${ }^{6}$ Department of Orthopaedics and Spine, Tokyo Neurological Center, 4-1-17 Toranomon, Minato-ku, Tokyo, Japan. ${ }^{7}$ Department of Orthopaedic Surgery, Faculty of Medicine, The University of Tokyo, 7-3-1 Hongo, Bunkyo-ku, Tokyo, Japan. ${ }^{8}$ These authors contributed equally: Teiji Harada, Toshiko lidaka, Hiroshi Yamada and Noriko Yoshimura. ${ }^{\circledR}$ email: t-takaya@wakayama-med.ac.jp
} 


\begin{tabular}{|c|c|c|}
\hline & Men & Women \\
\hline \multicolumn{3}{|c|}{ Age groups (years) } \\
\hline$\leq 49$ & $4 / 59(6.7 \%)$ & $0 / 117(0 \%)$ \\
\hline $50-59$ & $0 / 78(0 \%)$ & $0 / 186(0 \%)$ \\
\hline $60-69$ & $5 / 130(3.9 \%)$ & $5 / 292(1.7 \%)$ \\
\hline $70-79$ & $21 / 135(15.6 \%)$ & $11 / 278(4.0 \%)$ \\
\hline$\geq 80$ & $22 / 89(24.7 \%)$ & $0.0(4.3 \%)$ \\
\hline Total & $52 / 491(10.6 \%)$ & $0.0(2.1 \%)$ \\
\hline
\end{tabular}

Table 1. Prevalence of PGD by sex and five age groups. The prevalence of PGD tends to increase with age group in both sexes (Cochran-Armitage test; men: $\mathrm{p}<0.0001$, women: $\mathrm{p}=0.0004$ ). PGD pistol grip deformity.

\begin{tabular}{|c|c|c|c|}
\hline & PGD (+) & PGD (-) & p-value \\
\hline Number of participants (hips) & $73(102)$ & $1408(2860)$ & \\
\hline \multicolumn{4}{|l|}{ Demographics } \\
\hline Sex (men vs. women) & 52 vs. 21 & 439 vs. 969 & $<0.0001$ \\
\hline Age (years) & $75.3 \pm 11(72.6-77.9)$ & $64.8 \pm 12.8(64.1-65.5)$ & $<0.0001$ \\
\hline Height $(\mathrm{cm})$ & $157.4 \pm 9.8(155.1-159.7)$ & $156.2 \pm 9.1(155.7-156.7)$ & 0.2687 \\
\hline Weight (kg) & $59.5 \pm 11(56.9-62.1)$ & $56.3 \pm 11.2(55.7-56.9)$ & 0.0172 \\
\hline BMI $\left(\mathrm{kg} / \mathrm{m}^{2}\right)$ & $23.9 \pm 3.4(23.1-24.7)$ & $23.0 \pm 3.5(22.8-23.1)$ & 0.0231 \\
\hline Hip pain & $2 / 102(2.0 \%)$ & $56 / 2860(2.0 \%)$ & 0.9984 \\
\hline \multicolumn{4}{|l|}{ Hip parameters } \\
\hline $\mathrm{CE}$ angle $\left({ }^{\circ}\right)$ & $31.3 \pm 6.3(30.1-32.6)$ & $28.9 \pm 6.4(28.7-29.2)$ & $<0.0001$ \\
\hline mJSW (mm) & $4.0 \pm 0.9(3.8-4.2)$ & $3.9 \pm 0.8(3.9-3.9)$ & 0.5042 \\
\hline \multicolumn{4}{|l|}{ Spinopelvic parameters } \\
\hline $\operatorname{LL}\left(^{\circ}\right)$ & $40.8 \pm 18(36.5-44.9)$ & $45.7 \pm 13.3(45.0-46.4)$ & 0.0023 \\
\hline SS $\left(^{\circ}\right)$ & $28.8 \pm 11(26.3-31.2)$ & $31.9 \pm 9.0(31.4-32.4)$ & 0.0042 \\
\hline $\operatorname{PT}\left({ }^{\circ}\right)$ & $21.9 \pm 11(19.4-24.5)$ & $18.0 \pm 9.1(17.5-18.5)$ & 0.0003 \\
\hline $\operatorname{PI}\left({ }^{\circ}\right)$ & $50.7 \pm 12.5(47.8-53.6)$ & $49.9 \pm 10.6(49.4-50.5)$ & 0.5287 \\
\hline
\end{tabular}

Table 2. Comparison of the demographics between the groups with and without PGD. Prevalence of hip pain and hip parameters are calculated from 2962 hips. PGD pistol grip deformity, BMI body mass index, CE angle center-edge angle, $m J S W$ mean joint space width, $L L$ lumbar lordosis, $S S$ sacral slope, $P T$ pelvic tilt, $P I$ pelvic incidence. Data are means \pm standard deviation ( $95 \%$ confidence interval). Bold values indicate statistically significant differences between the two groups.

\section{Results}

Prevalence of pistol grip deformity. Table 1 shows the prevalence of PGD in the overall population and the subgroups of age, classified by sex. In the overall population, the prevalence of PGD was 73/1481 (4.9\%). The prevalence of PGD in men $(52 / 491 ; 10.6 \%)$ was significantly higher than that in women $(21 / 990 ; 2.1 \%)$. The risk ratio for men was 4.99 (95\% confidence interval (CI) 3.04-8.19), which was higher than that for women. Moreover, the prevalence of PGD significantly increased with age for both sexes (Supplementary Figure S1).

Comparison of the demographics between the groups with and without pistol grip deformity. The PGD-positive group had a significantly higher proportion of men, older age, and higher body weight and body mass index (BMI) than the PGD-negative group (Table 2).

Association of pistol grip deformity with hip pain. Among the 102 PGD-positive hips, only two participants (2.0\%) experienced hip pain on the affected side. No significant association was observed between PGD and hip pain (risk ratio 1.0 [95\% CI 0.97-1.03]) (Table 2).

Association of pistol grip deformity with hip parameters. The PGD-positive group had a significantly larger center edge (CE) angle than the PGD-negative group (Table 2). However, multiple logistic regression analysis, including sex, age, BMI, CE angle, and medial joint space width (mJSW) as explanatory variables, revealed that the presence of PGD was not significantly associated with CE or mJSW. Male sex, age, and BMI were significantly associated with the presence of PGD in this model (Table 3).

Association of pistol grip deformity with spino-pelvic parameters. Regarding the comparison of spino-pelvic parameters, the lumbar lordosis (LL), sacral slope (SS), and pelvic tilt (PT) were found to be signifi- 


\begin{tabular}{|l|l|l|l|c|}
\hline & Reference & Odds ratio & $\mathbf{9 5 \%}$ CI & p-value \\
\hline Sex & Men vs. women & $\mathbf{4 . 5 4}$ & $\mathbf{2 . 8 5 - 7 . 2 5}$ & $<\mathbf{0 . 0 0 0 1}$ \\
\hline Age & $+\mathbf{1}$ year & $\mathbf{1 . 0 9}$ & $\mathbf{1 . 0 7 - 1 . 1 2}$ & $<\mathbf{0 . 0 0 0 1}$ \\
\hline BMI & $+\mathbf{1 ~ k g / \mathbf { m } ^ { 2 }}$ & $\mathbf{1 . 1 2}$ & $\mathbf{1 . 0 6}-\mathbf{1 . 1 9}$ & $\mathbf{0 . 0 0 0 2}$ \\
\hline CE angle & $+1^{\circ}$ & 1.03 & $0.99-1.07$ & 0.1252 \\
\hline mJSW & $+1 \mathrm{~mm}$ & 0.91 & $0.68-1.22$ & 0.537 \\
\hline
\end{tabular}

Table 3. Association of PGD with hip parameters (multivariate analysis). Odds ratios are calculated by multiple logistic regression analysis on 2962 hips. PGD pistol grip deformity, 95\% CI 95\% confidence interval, $B M I$ body mass index, $C E$ angle center-edge angle, $m J S W$ mean joint space width. Bold values indicate a statistically significant association with PGD.

\begin{tabular}{|l|l|l|l|l|}
\hline & Reference & Odds ratio & $\mathbf{9 5 \%} \mathbf{C I}$ & p-value \\
\hline LL & $+1^{\circ}$ & 1.00 & $0.97-1.03$ & 0.7812 \\
\hline PT & $+1^{\circ}$ & 1.04 & $\mathbf{1 . 0 0 - 1 . 1 0}$ & 0.0746 \\
\hline PI & $+1^{\circ}$ & 0.99 & $0.95-1.04$ & 0.8963 \\
\hline
\end{tabular}

Table 4. Association of PGD with spino-pelvic parameters (multivariate analysis). Bold values indicate a positive association with PGD. Odds ratios are calculated by multiple logistic regression analysis after adjustment by age, sex and body mass index on 1481 participants. In creating the model, the propensity score for the objective variable is calculated from the adjusted variables and treated as one variable. $P G D$ pistol grip deformity, 95\% CI 95\% confidence interval, $L L$ lumbar lordosis, $P T$ pelvic tilt, $P I$ pelvic incidence.

cantly different between the groups with and without PGD. Pelvic incidence (PI) was not significantly different between the two groups (Table 3). Subsequently, we conducted a multiple logistic regression analysis wherein the LL, PT, and PI served as explanatory variables, and age, sex, and BMI served as adjusted variables. SS was not included in this model to avoid multicollinearity because we used the algorithm "PI = PT + SS." Moreover, the propensity score for the objective variable was calculated from the adjusted variables and treated as one variable to avoid overfitting. The presence of PGD was not significantly associated with any of the spino-pelvic parameters; however, PT demonstrated a positive odds ratio (95\% CI 1.00-1.10) (Table 4).

\section{Discussion}

The most notable result of this study was that the prevalence of PGD increased with age in both men and women. In addition, PGD was significantly associated with weight and BMI. These findings may suggest that the etiology of PGD involves acquired factors in addition to congenital morphological anomalies of the femoral neck, at least in the Japanese population.

In this study, the overall prevalence of PGD was $4.9 \%$ (10.6\% in men and $2.1 \%$ in women). To the best of our knowledge, this is the first study to successfully elucidate the epidemiological indices of PGD and the related factors in a large (approximately 1500) Asian population. A previous study by Goodman et al. examined 2665 cadavers and found 215 cadavers with PGD $(8 \%)^{14}$. In another study by Doherty et al., the overall prevalence of PGD was $3.61 \%$ (6.37\% in men and $0.39 \%$ in women $)^{15}$. That study included people who had been in the hospital for intravenous urography, with no radiographic hip osteoarthritis. Consistent with their findings, our results suggested that the prevalence of PGD in the Asian participants (Japanese) was relatively lower than that in Europe and the United States. Similar to our results, several previous studies have reported that PGD is more common in men than in women ${ }^{16,17}$. Goodman et al. have reported 122 cadavers with a bilateral PGD incidence of $56.7 \%{ }^{14}$, which is considerably high, similar to that in our study $(29 / 71 ; 40.8 \%)$.

Interestingly, the most notable result of this study was that the prevalence of PGD increased with age in both men and women. No past studies have reported a correlation between the prevalence of PGD and age; rather, it has been reported that the prevalence of PGD in men tended to reduce with age ${ }^{15}$. Allen et al. have examined 113 cases with cam-type FAI and reported that a deformity of the head-neck junction could be congenital ${ }^{18}$. However, our findings suggest that the etiology of PGD involves acquired factors in addition to congenital morphological anomalies of the femoral neck in the Japanese population. Only racial differences could cause variations in the correlation of age with PGD. However, regarding the relationship between PGD and spino-pelvic parameters, only PT showed a possible correlation with PGD in our study. An increase in PT indicates pelvic retroversion with age. Thus, instability due to decreased coverage of the antero-lateral femoral head, resulting from pelvic retroversion between the acetabulum and femoral head, might result in PGD as a physiological response.

Another notable result of this study was that the presence of PGD alone was not correlated with either hip pain or joint space narrowing. Based on previous studies from Western countries, PGD is considered susceptible to impingement between the anterior femoral neck and the adjacent acetabulum, which is a common cause of hip pain, labral tears, and osteoarthritis ${ }^{5,15,18-20}$. The difference between our study results and those of previous reports is probably due to the differences in hip joint morphology between Japanese and Western people. Nakamura et al. have evaluated 254 normal hip joints on radiographs and reported that the acetabular roofs of Japanese people are shallower than those of Europeans and Americans ${ }^{21}$. Moreover, Takeyama et al. reported that the anteroposterior 
diameter of the femoral neck is smaller in the Japanese than in Europeans and Americans, and an impingement between the anterior femoral neck and the adjacent acetabulum is less likely in this population ${ }^{22}$. Our findings suggest that the pathological significance of PGD differs between Japan and Western countries. We should consider that this study excluded patients who had hip osteoarthritis (Kellgren-Lawrence grade $\geq 3$ ) and those who had undergone total hip arthroplasty or bilateral hip arthroplasty. Symptoms might not necessarily appear until PGD progresses to hip osteoarthritis. However, historically, Takeyama et al. retrospectively investigated 946 hip joints of patients who underwent hip surgery and reported the prevalence of characteristic findings of FAI $(\fallingdotseq P G D)$ to be $0.6 \%$ in Japan ${ }^{22}$. The actual prevalence of FAI and associated factors, such as hip pain, remain unclear because only a few epidemiological studies on PGD have been conducted in Japan.

This study had several limitations. First, causal relationships between the evaluated variables were not determined because of the cross-sectional study design. Follow-up with a longitudinal study is needed in this cohort. Second, a selection bias might have existed. Among the participants, the proportion of elderly individuals was high because the Research on Osteoarthritis/Osteoporosis Against Disability (ROAD) study was instituted to elucidate the epidemiology of degenerative diseases of locomotive organs. Moreover, regional selection bias should be considered because the subjects (voluntary participants) were recruited from only two regions. However, Yoshimura et al. reported that the participants of the ROAD study are considered representative of the Japanese population $^{23}$. Thus, these findings may not be generalizable to other populations. Applying the results of this study to different races or countries with different lifestyles would require careful judgment. In the future, we will prospectively investigate the percentage of PGD-positive patients who gradually develop osteoarthritis of the hip and factors affecting the transition from PGD to osteoarthritis of the hip using subsequent ROAD study data.

This study clarified the epidemiological index of PGD and related factors in a general population of Japan. PGD has been considered a congenital morphological variation. However, the results of this study indicate that several acquired factors can be associated with PGD, at least in Japanese people, because the prevalence of PGD increases with age. Moreover, PGD was not significantly associated with hip pain. Although the study is limited by its cross-sectional design and potential selection bias, we believe our results provide new insights into the etiology and clinical significance of PGD.

\section{Materials and methods}

Participants. The ROAD study is a nationwide prospective study of bone and joint diseases comprising population-based cohorts from several communities in Japan. The representative bone and joint diseases of the ROAD study are osteoarthritis and osteoporosis. A detailed profile of the ROAD cohort has been previously reported $^{23-28}$. Briefly, the participants in this study included those recruited during the 3rd examination of the ROAD study, which was conducted from October 2012 to December 2013. In addition to the previous participants, inhabitants of the mountainous area in Hidakagawa (with a population of 11,300 people $/ 330 \mathrm{~km}^{2}$ ) and coastal areas in Taiji $\left(3,500\right.$ people $\left./ 6 \mathrm{~km}^{2}\right)$ in the Wakayama prefecture $\left(987,500\right.$ people $\left./ 4725 \mathrm{~km}^{2}\right)$ of Japan who were willing to participate in the ROAD survey were also included during this 3rd examination. Overall, 1575 individuals (513 men, 1062 women) participated in the 3rd examination of the ROAD study. Of these participants, 20 (1.3\%) did not undergo plain radiography, $7(0.4 \%)$ could not read, $12(0.8 \%)$ had proximal femur fracture, $10(0.6 \%)$ had undergone total hip arthroplasty or bilateral hip arthroplasty, and $2(0.1 \%)$ had hip osteoarthritis (Kellgren-Lawrence grade $\geq 3$ ). In total, 94 individuals, including 43 participants (2.7\%) with incomplete spine or hip data, were excluded. The remaining 2962 hips of 1481 participants (94.0\%) aged 19-94 years (mean 65.3 years) were included in this study (Fig. 1). The characteristics of the participants are shown in Table 5.

Radiographs of the pelvis, including the hip joints and lumbar spine, were available for the 1481 participants (491 men, 990 women).

The participants completed an interviewer-administered questionnaire, which included questions on family history, medical history, and previous history of hip injury. Anthropometric measurements included height and weight, from which the BMI (weight $[\mathrm{kg}] /$ height $\left[\mathrm{m}^{2}\right]$ ) was calculated. Furthermore, all participants were interviewed by well-experienced orthopedists regarding pain in both hips. The orthopedists asked, "Have you experienced right hip pain on most days in the past month, in addition to now?" and "Have you experienced left hip pain on most days in the past month, in addition to now?" Participants who answered "yes" were considered to have hip pain.

Radiographic evaluation. All participants underwent radiographic examination of both hips using the anteroposterior view with weight-bearing and the feet internally rotated. Fluoroscopic guidance with a horizontal anteroposterior X-ray beam was used to properly visualize the joint space. The hip radiographs were read by three well-experienced orthopedic surgeons who were blinded to the participant's clinical status. The following parameters were measured: existence of $\mathrm{PGD}^{20,29}, \mathrm{CE}$ angle, and $\mathrm{mJSW}^{30}$. Based on past reports by Tannast et al. ${ }^{31}$ and Fukushima et al. ${ }^{32}$, we defined PGD as a prominent lateral offset of the femoral head-neck junction. By drawing a circle around the femoral head on the anteroposterior view, the overhang was measured. Instances wherein the femoral head overhang was small were similarly considered PGD-positive cases (Fig. 2).

To evaluate the intra-observer variability of the hip parameters, 50 randomly selected radiographs of the hip were analyzed by the same observer $>1$ month after the first reading. Additionally, 50 other radiographs were analyzed by the three orthopedic surgeons using the same atlas for interobserver variability. The intra-class and inter-class correlation coefficients for the CE angle were 0.94 and 0.85 , respectively, and 0.97 and 0.97 , respectively, for $\mathrm{mJSW}$. The interobserver variability for the center of the femoral head was confirmed by kappa analysis to be sufficient for assessment $(\kappa=1)$.

Moreover, for spino-pelvic alignment parameters, standing lateral radiographs of the whole spine and pelvis were taken. Each radiograph was aligned such that the edge of the film was the reference for vertical alignment. 


\section{Selection of participants}

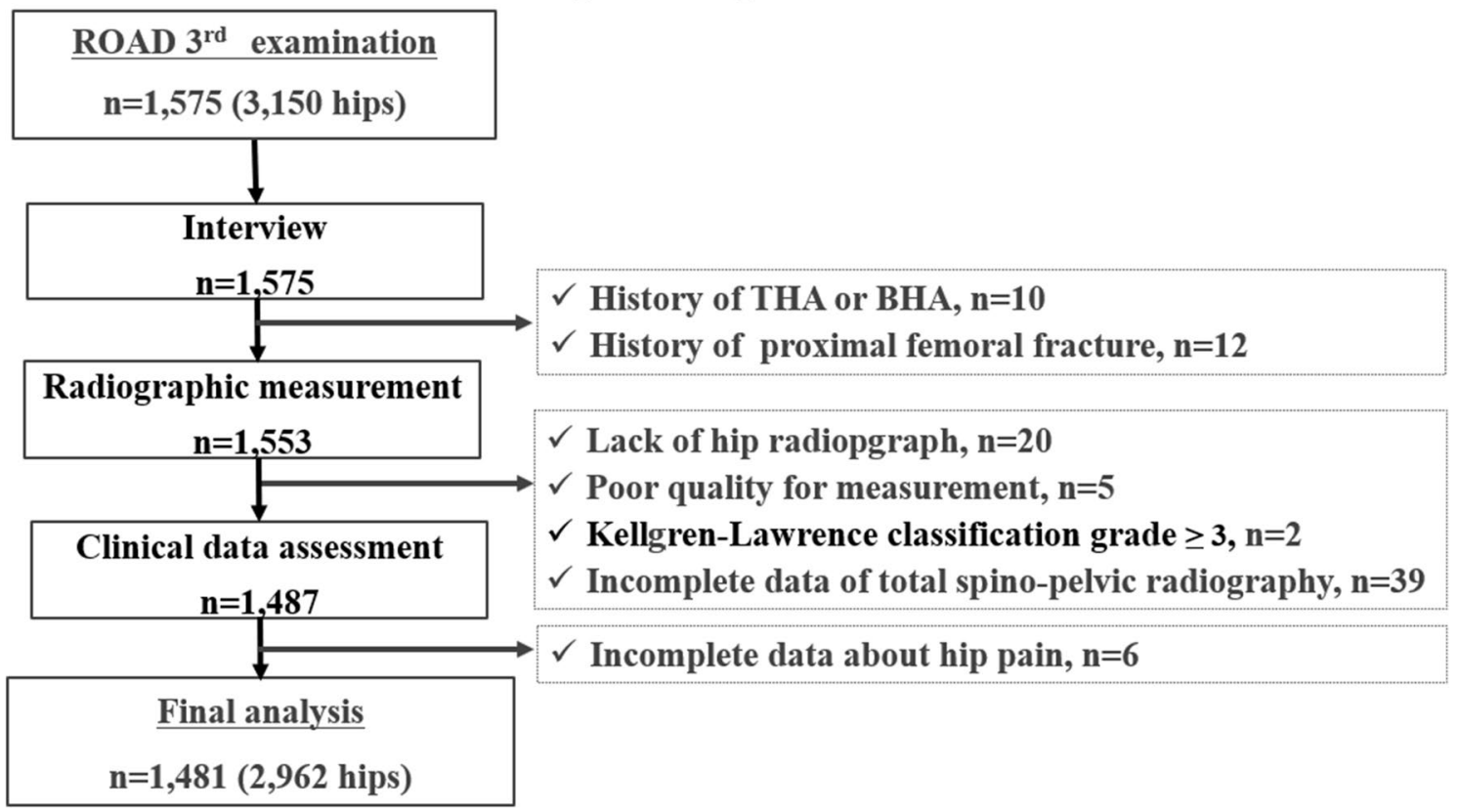

Figure 1. Flow diagram depicting the study enrollment strategy. Participants in the study are recruited from the residents who participated in the 2012 and 2013 examinations for clinical evaluation, as a part of the Research on Osteoarthritis/Osteoporosis Against Disability (ROAD) study. THA, total hip arthroplasty; BHA, bipolar hip arthroplasty.

\begin{tabular}{|l|l|l|l|}
\hline \multicolumn{2}{|l|}{ Total } & Men & Women \\
\hline Number of participants (hips) & $1481(2962)$ & $491(982)$ & $990(1980)$ \\
\hline Demographics & $65.3 \pm 13.0(64.7-66.0)$ & $66.2 \pm 13.8(65.0-67.4)$ & $64.9 \pm 12.5(64.1-65.7)$ \\
\hline Age (years) & $156.3 \pm 9.1(155.8-156.7)$ & $164.8 \pm 7.2(164.2-165.5)$ & $152.0 \pm 6.6(151.6-152.4)$ \\
\hline Height $(\mathrm{cm})$ & $56.4 \pm 11.3(55.8-57.0)$ & $64.6 \pm 11.4(63.6-65.6)$ & $52.4 \pm 8.7(51.8-52.9)$ \\
\hline Weight $(\mathrm{kg})$ & $23 \pm 3.5(22.8-23.2)$ & $23.7 \pm 3.5(23.4-24.0)$ & $22.7 \pm 3.5(22.4-22.9)$ \\
\hline BMI $\left(\mathrm{kg} / \mathrm{m}^{2}\right)$ & $29.0 \pm 6.4(28.8-29.3)$ & $29.9 \pm 6.0(29.6-30.3)$ & $28.6 \pm 6.5(28.3-28.9)$ \\
\hline Hip parameters & $3.9 \pm 0.8(3.9-3.9)$ & $4.2 \pm 0.8(4.1-4.2)$ & $3.8 \pm 0.7(3.7-3.8)$ \\
\hline CE Angle $\left(^{\circ}\right)$ & $45.4 \pm 13.7(44.7-46.1)$ & $44.1 \pm 12.9(43.0-45.3)$ & $46.1 \pm 14.0(45.2-47.0)$ \\
\hline mJSW (mm) & $31.8 \pm 9.1(31.3-32.2)$ & $31.5 \pm 8.7(30.8-32.3)$ & $31.9 \pm 9.4(31.3-32.5)$ \\
\hline Spinopelvic parameters & $18.2 \pm 9.2(17.7-18.7)$ & $15.6 \pm 7.8(14.9-16.3)$ & $19.5 \pm 9.6(18.9-20.0)$ \\
\hline LL $\left(^{\circ}\right)$ & $49.9 \pm 10.7(49.4-50.5)$ & $47.2 \pm 9.9(46.3-48.0)$ & $51.3 \pm 10.8(50.7-52.0)$ \\
\hline SS $\left(^{\circ}\right)$ & PT $\left(^{\circ}\right)$ & & \\
\hline PI $\left(^{\circ}\right)$ &
\end{tabular}

Table 5. Characteristics of the participants. Data are means \pm standard deviation $(95 \%$ confidence interval of the mean). Hip parameters were calculated from 2962 hips. BMI body mass index, $L L$ lumbar lordosis, $S$ sacral slope, $P$ T pelvic tilt, PI pelvic incidence.

On the standing lateral radiographs, the following parameters were measured: LL (the Cobb angle from the upper endplate of L1 to the lower endplate of S1), SS (the angle between the tangent line to the superior endplate of S1 and the horizontal plane), PT (the angle between the line connecting the midpoint of the sacral plate to the axes of the femoral heads and the vertical axis), and PI (the angle between the line perpendicular to the sacral plate at its midpoint and the line connecting this point to the axes of the femoral heads) (Fig. 3$)^{33,34}$.

Statistical analysis. Statistical analyses were performed using JMP (version 14; SAS Institute Inc., Cary, NC). Continuous variables are expressed as the mean \pm standard deviation (SD) and 95\% CIs. The participants were classified into five age groups based on birth-year decade: (1) less than 50 years, (2) 50-59 years, (3) 60-69 years, (4) 70-79 years, and (5) 80 years and older. The Cochran-Armitage test was conducted to determine the trend of PGD prevalence among the five age groups. Differences in age, height, weight, BMI, hip parameters, 


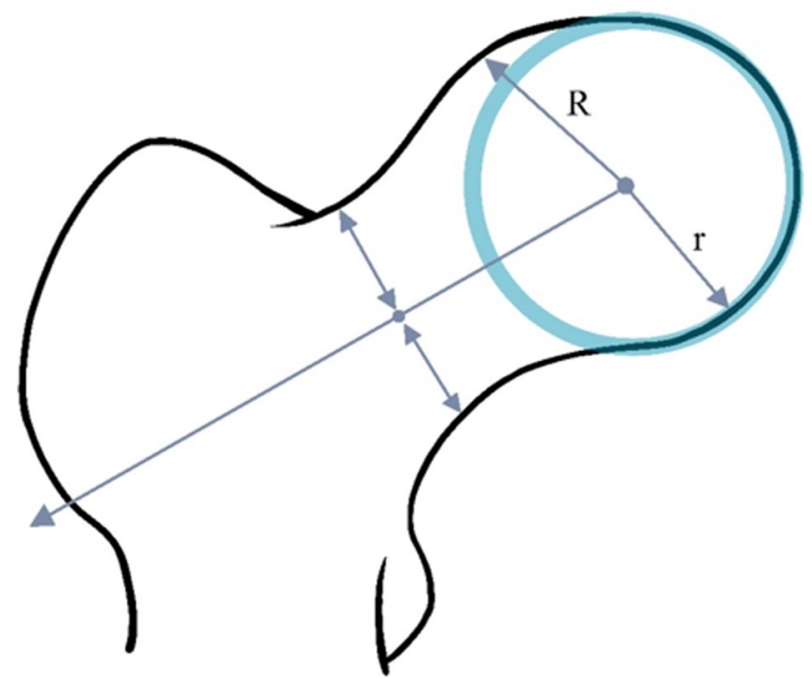

Figure 2. Schema of the definition of a PGD-positive case. The overhang is measured by drawing a circle around the femoral head on the anteroposterior view. Even a small overhang of the femoral head $(\mathrm{R}>\mathrm{r})$ is considered a PGD-positive case. PGD, pistol grip deformity.

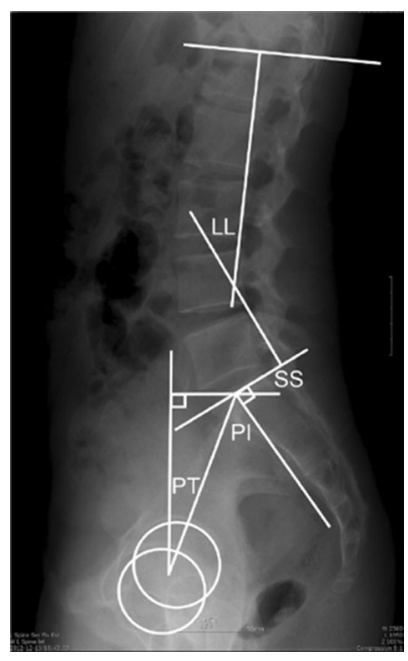

Figure 3. Schema of the measuring methods for each spino-pelvic parameter on a standing radiograph of the whole spine and pelvis. LL, lumbar lordosis; SS, sacral slope; PI, pelvic incidence; PT, pelvic tilt.

and spino-pelvic parameters were examined between men and women or between the PGD-positive and PGDnegative groups using a non-paired t-test. A chi-square test was conducted to compare the prevalence of PGD between men and women or between the groups (with versus without hip pain). The association of the variables, including age, BMI, sex, spino-pelvic parameters, and hip parameters, with the presence/absence of PGD was examined via multiple logistic regression analysis. Risk ratios or odds ratios are provided with $95 \%$ CIs.

Ethics declarations. All participants provided written informed consent for their participation and for the publication of the study in print and in electronic form.

The study was conducted with the approval of the appropriate ethics committees. The establishment of a cohort related to this study has been approved by the Research Ethics Review Committee of the University of Tokyo (No. 1326). The procedures followed were in accordance with the ethical standards of the responsible committee on human experimentation (institutional and national) and with the Helsinki Declaration of 1975, as revised in 2000 .

\section{Data availability}

All data generated or analyzed during this study are available from the corresponding author upon reasonable request. 
Received: 6 October 2020; Accepted: 2 March 2021

Published online: 16 March 2021

\section{References}

1. Murray, R. O. The aetiology of primary osteoarthritis of the hip. Br. J. Radiol. 38, 810-824 (1965).

2. Stulberg, S.D. Unrecognized childhood hip disease a major cause of idiopathic osteoarthritis of the hip. In The Hip, Proceedings of the Third Open Scientific Meeting of the Hip Society (eds. Cordell, L.D. et al.) 212-228 (St. Louis MO CV Mosby1975).

3. Myers, S. R., Eijer, H. \& Ganz, R. Anterior femoroacetabular impingement after periacetabular osteotomy. Clin. Orthop. Relat. Res. 363, 93-99 (1999).

4. Leunig, M. et al. Slipped capital femoral epiphysis: Early mechanical damage to the acetabular cartilage by a prominent femoral metaphysis. Acta Orthop. Scand. 71, 370-375 (2000).

5. Ganz, R. et al. Femoroacetabular impingement: A cause for osteoarthritis of the hip. Clin. Orthop. Relat. Res. 417, 112-120 (2003).

6. Beck, M. et al. Anterior femoroacetabular impingement: part II. Midterm results of surgical treatment. Clin. Orthop. Relat. Res. 481, 67-73 (2004).

7. Agricola, R. et al. Cam impingement causes osteoarthritis of the hip: A nationwide prospective cohort study (CHECK). Ann. Rheum. Dis. 72, 918-923 (2013).

8. Reynolds, D., Lucas, J. \& Klaue, K. Retroversion of the acetabulum. A cause of hip pain. J. Bone Joint Surg. Br. 81, 281-288 (1999).

9. Ganz, R., Leunig, M., Leunig-Ganz, K. \& Harris, W. H. The etiology of osteoarthritis of the hip: An integrated mechanical concept. Clin. Orthop. Relat. Res. 466, 264-272 (2008).

10. Aprato, A. et al. Magnetic resonance arthrography for femoroacetabular impingement surgery: Is it reliable?. J. Orthop. Traumatol. 14, 201-206 (2013).

11. Weinberg, D. S., Gebhart, J. J., Liu, R. W. \& Salata, M. J. Radiographic signs off femoroacetabular impingement are associated with decreased pelvic incidence. Arthroscopy 32, 806-813 (2016).

12. Gebhart, J. J., Weinberg, D. S., Bohl, M. S. \& Liu, R. W. Relationship between pelvic incidence and osteoarthritis of the hip. Bone Joint Res. 5, 66-72 (2016).

13. Clarke, M. T., Arora, A. \& Villar, R. N. Hip arthroscopy: Complications in 1054 cases. Clin. Orthop. Relat. Res. 406, 84-88 (2003).

14. Goodman, D. A. et al. Subclinical slipped capital femoral epiphysis. Relationship to osteoarthrosis of the hip. J. Bone Joint Surg. Am. 79, 1489-1497 (1997).

15. Doherty, M. et al. Nonspherical femoral head shape (pistol grip deformity), neck shaft angle, and risk of hip osteoarthritis: A case-control study. Arthritis Rheum. 58, 3172-3182 (2008).

16. Laborie, L. B. et al. Prevalence of radiographic findings thought to be associated with femoroacetabular impingement in a population-based cohort of 2081 healthy young adults. Radiology 260, 494-502 (2011).

17. Lung, R. et al. The prevalence of radiographic femoroacetabular impingement in younger individuals undergoing total hip replacement for osteoarthritis. Clin. Rheumatol. 31, 1239-1242 (2012).

18. Allen, D., Beaulé, P. E., Ramadan, O. \& Doucette, S. Prevalence of associated deformities and hip pain in patients with cam-type femoroacetabular impingement. J. Bone Joint Surg. Br. 91, 589-594 (2009).

19. Wisniewski, S. J. \& Grogg, B. Femoroacetabular impingement: An overlooked cause of hip pain. Am. J. Phys. Med. Rehabil. 85, 546-549 (2006)

20. Tanzer, M. \& Noiseux, N. Osseous abnormalities and early osteoarthritis: The role of hip impingement. Clin. Orthop. Relat. Res. 429, 170-177 (2004)

21. Nakamura, S., Ninomiya, S. \& Nakamura, T. Primary osteoarthritis of the hip joint in Japan. Clin. Orthop. Relat. Res. 241, 190-196 (1989).

22. Takeyama, A., Naito, M., Shiramizu, K. \& Kiyama, T. Prevalence of femoroacetabular impingement in Asian patients with osteoarthritis of the hip. Int. Orthop. 33, 1229-1232 (2009).

23. Yoshimura, N. et al. Cohort profile: Research on osteoarthritis/osteoporosis against disability study. Int. J. Epidemiol. 39, 988-995 (2010).

24. Oka, H. et al. Normal and threshold values of radiographic parameters for knee osteoarthritis using a computer-assisted measuring system (KOACAD): The ROAD study. J. Orthop. Sci. 15, 781-789 (2010).

25. Muraki, S. et al. Prevalence of radiographic knee osteoarthritis and its association with knee pain in the elderly of Japanese population-based cohorts: The ROAD study. Osteoarthr. Cartil. 17, 1137-1143 (2009).

26. Yoshimura, N. et al. Prevalence of knee osteoarthritis, lumbar spondylosis, and osteoporosis in Japanese men and women: The research on osteoarthritis/osteoporosis against disability study. J. Bone Miner. Metab. 27, 620-628 (2009).

27. Iidaka, T. et al. Prevalence of radiographic hip osteoarthritis and its association with hip pain in Japanese men and women: The ROAD study. Osteoarthr. Cartil. 24, 117-123 (2016).

28. Iidaka, T. et al. Radiographic measurements of the hip joint and their associations with hip pain in Japanese men and women: The Research on Osteoarthritis/osteoporosis Against Disability (ROAD) study. Osteoarthr. Cartil. 25, 2072-2079 (2017).

29. Castañeda, P., Ponce, C., Villareal, G. \& Vidal, C. The natural history of osteoarthritis after a slipped capital femoral epiphysis/the pistol grip deformity. J. Pediatr. Orthop. 33(Suppl 1), S76-82 (2013).

30. Conrozier, T. et al. Quantitative measurement of joint space narrowing progression in hip osteoarthritis: A longitudinal retrospective study of patients treated by total hip arthroplasty. Br. J. Rheumatol. 37, 961-968 (1998).

31. Tannast, M., Siebenrock, K. A. \& Anderson, S. E. Femoroacetabular impingement: Radiographic diagnosis-what the radiologist should know. AJR Am. J. Roentgenol. 188, 1540-1552 (2007).

32. Fukushima, K. et al. Prevalence of radiographic findings of femoroacetabular impingement in the Japanese population. J. Orthop. Surg. Res. 9, 25 (2014).

33. Schwab, F., Patel, A., Ungar, B., Farcy, J. P. \& Lafage, V. Adult spinal deformity-postoperative standing imbalance: How much can you tolerate? An overview of key parameters in assessing alignment and planning corrective surgery. Spine 35, 2224-2231 (2010).

34. Asai, Y. et al. Sagittal spino-pelvic alignment in adults: The Wakayama Spine Study. PLoS ONE 12, e0178697. https://doi. org/10.1371/journal.pone.0178697 (2017).

\section{Acknowledgements}

The authors wish to thank Dr. Naoki Hirabayashi of the Kawakami Clinic in the town of Hidakagawa; Mrs. Tomoko Takajiri, Mrs. Rie Takiguchi, Mrs. Kyoko Maeda, and other members of the town office in Hidakagawa Town; Dr. Shinji Matsuda of the Shingu Public Health Center; and Mrs. Tamako Tsutsumi, Mrs. Kanami Maeda, Mrs. Megumi Takino, Mrs. Shuko Okada, Mrs. Kazuyo Setoh, Mrs. Chise Ryouno, Mrs. Miki Shimosaki, Mrs. Chika Yamaguchi, Mrs. Yuki Shimoji, and the other members of the town office in the town of Taiji for their assistance in locating and scheduling participants for examinations. 


\section{Author contributions}

Conception and design of the study: T.T., H.H., K.N., H.K., M.Y., S.T., H.Y., and N.Y. Analysis and interpretation of data: T.T., H.H., W.T., and H.O. Collection and assembly of data: T.T., T.H., T.I., H.H., Y.A., S.M., T.A., and N.Y. Drafting of the article: T.T., and W.T. Critical revision of the article for important intellectual content: T.T., H.H., N.Y., and H.Y. Final approval of article: T.T., H.H., N.Y., and H.Y. T.T. takes responsibility for the integrity of the work in its entirety.

\section{Funding}

This work was supported by a Grant-in-Aid for H17-Men-eki-009 (Director, Kozo Nakamura), H20-Choujyu-009 (Director, Noriko Yoshimura), H23-Choujyu-002 (Director, Toru Akune), H25-Choujyu-007 (Director, Noriko Yoshimura), and H25-Nanchitou (Men)-005 (Director, Sakae Tanaka) of the Ministry of Health, Labour and Welfare. Support was also received from Scientific Research, 24659317 (to Noriko Yoshimura); B23390356, C20591774, and Challenging Exploratory Research 23659580 (to Shigeyuki Muraki); Challenging Exploratory Research 24659666 and 21659349, and Young Scientist A18689031 (to Hiroyuki Oka); B23390357 and C20591737 (to Toru Akune); and Collaborating Research with NSF 08033011-00262 (Director, Noriko Yoshimura); from the Ministry of Education, Culture, Sports, Science and Technology in the Japan Osteoporosis Society (2006-1), (Noriko Yoshimura, Shigeyuki Muraki, Hiroyuki Oka, and Toru Akune). Finally, a research aid was obtained from the Japanese Orthopedic Association (JOA-Subsidized Science Project Research 2006-1 and 2010-2; Director, Hiroshi Kawaguchi). The study sponsors played no role in the study design; the collection, analysis, and interpretation of data; the writing of the report; or the decision to submit the paper for publication.

\section{Competing interests}

The authors declare no competing interests.

\section{Additional information}

Supplementary Information The online version contains supplementary material available at https://doi. org/10.1038/s41598-021-85521-X.

Correspondence and requests for materials should be addressed to T.T.

Reprints and permissions information is available at www.nature.com/reprints.

Publisher's note Springer Nature remains neutral with regard to jurisdictional claims in published maps and institutional affiliations.

Open Access This article is licensed under a Creative Commons Attribution 4.0 International License, which permits use, sharing, adaptation, distribution and reproduction in any medium or format, as long as you give appropriate credit to the original author(s) and the source, provide a link to the Creative Commons licence, and indicate if changes were made. The images or other third party material in this article are included in the article's Creative Commons licence, unless indicated otherwise in a credit line to the material. If material is not included in the article's Creative Commons licence and your intended use is not permitted by statutory regulation or exceeds the permitted use, you will need to obtain permission directly from the copyright holder. To view a copy of this licence, visit http://creativecommons.org/licenses/by/4.0/.

(C) The Author(s) 2021 